\title{
Software y bibliografía para enseñanza de interpretación de datos en una asignatura de Máster
}

\author{
M.Carmen Martínez-Bisbal ${ }^{\text {a,b,c,d }}{ }^{*}$, Rafael Masot Peris ${ }^{\text {a,e }}$, Miguel Alcañiz Fillol a,e
}

\begin{abstract}
anstituto Interuniversitario de Investigación de Reconocimiento Molecular y Desarrollo Tecnológico, Universitat Politècnica de València, Valencia, Camino de Vera s/n, 46022 Valencia, mamarbis@upvnet.upv.es, bDepartamento de Química-Física, Universitat de València, C/Doctor Moliner 50, 46100 Burjasot, Valencia, 'Unidad Mixta de Investigación en Nanomedicina y Sensores, Universitat Politècnica de València - IIS La Fe, Av. Fernando Abril Martorell, 106 Torre A $6^{\mathrm{a}}$ planta. 46026 Valencia, ${ }^{\mathrm{d}} \mathrm{CIBER}$ de Bioingeniería, Biomateriales y Nanomedicina (CIBER-BBN), Instituto de Salud Carlos III, ${ }^{e}$ Departamento de Ingeniería Electrónica, Escuela Técnica Superior de Ingeniería del Diseño, Universitat Politècnica de València, Valencia, Camino de Vera s/n, 46022 Valencia mialcan@upvnet.upv.es, $\underline{\text { ramape@eln.upv.es }}$
\end{abstract}

\begin{abstract}
In the Master's Degree in Sensors for Industrial Applications of the Universitat Politècnica de València, the subject "Modeling techniques" is taught. "Application of multivariate techniques to real experiences" is one of the 3 didactic units that make up this subject. This unit focuses on the one hand on the explanation of the philosophy of electronic tongues as an example of sensors in the industrial field, and on the other hand, on the use of multivariate statistics for interpretation of the information of these sensors. The teaching of this didactic unit is carried out with an intuitive and simple software such as the SOLO program (Eigenvector Research, Inc.) and its application to a set of data derived from experiments carried out previously by the teacher's research team. As proof of mastery of the subject the students are required to reproduce the process in a new set of data provided by the teacher and obtained also in the laboratory by the teacher's research team. For both sets of data there are the respective research articles in which the student can consult a greater detail of the approach of the work and the discussion of the results.
\end{abstract}

Keywords: Methodology, ICT, dynamization

\section{Resumen}

En el Máster Universitario de Sensores para Aplicaciones Industriales de la Universitat Politècnica de València se imparte la asignatura "Técnicas de modelización". "Aplicación de técnicas multivariantes a experiencias reales" es una de las 3 unidades didácticas que integran esta asignatura. Esta unidad se centra en por una parte en la explicación de la filosofía de las lenguas electrónicas como ejemplo de sensores en el ámbito industrial y por otra, en el uso de la estadística multivariante para interpretación de la información de estos sensores. La docencia de esta unidad didáctica se realiza con un software intuitivo y sencillo como es el programa SOLO (Eigenvector Research, Inc.) y su aplicación a un conjunto de datos derivados de experimentos llevados a 
cabo previamente por el equipo de investigación del docente. Como comprobación del dominio de la materia a los alumnos se les requiere la reproducción del proceso en un nuevo conjunto de datos proporcionado por el profesor y obtenido también en el laboratorio por el equipo de investigación del docente. Para ambos conjuntos de datos existen los respectivos artículos de investigación en los que el alumno puede consultar un mayor detalle del planteamiento del trabajo y la discusión de los resultados.

Palabras clave: Metodología, TIC, dinamización

\section{Introducción}

En el "Máster Universitario de Sensores para Aplicaciones Industriales" de la Universitat Politècnica de València se imparte la asignatura obligatoria "Técnicas de modelización" dentro de la materia "Herramientas para el diseño y desarrollo de sensores". Esta asignatura se imparte en el primer cuatrimestre y ocupa 4.5 créditos. "Aplicación de técnicas multivariantes a experiencias reales" es una de las 3 unidades didácticas que integran esta asignatura. Esta unidad didáctica tiene como objetivos por una parte el acercamiento de la filosofía de las lenguas electrónicas al alumnado, siendo las lenguas electrónicas un ejemplo de sensores para uso en el ámbito industrial y por otra parte, en la introducción en el uso de la estadística multivariante para la extracción e interpretación de la información de estos sensores.

En la industria, resulta conveniente disponer de instrumentos de bajo coste, respuesta rápida y de lectura rápida para la monitorización de la producción. En ese sentido, las técnicas electroquímicas son la base de un amplio número de estrategias analíticas de bajo coste y respuesta rápida. Las lenguas electrónicas son sistemas analíticos basados en sensores de baja selectividad y sensibilidad cruzada que permiten la clasificación de muestras complejas para la caracterización de sus parámetros fisicoquímicos (Winquist, 1997). Las lenguas electrónicas pueden clasificarse según la técnica de detección en la que están basadas como pueden ser la potenciometría, voltametría, colorimetría, ondas acústicas y otros. Las lenguas electrónicas basadas en voltamperometría han sido investigados intensivamente en los últimos años debido a su alta sensibilidad y elevada relación señal/ruido. En este tipo de lenguas electrónicas se aplica un conjunto de pulsos de potencial a diferentes electrodos metálicos y se mide el paso de corriente resultante. Estas lenguas tienen aplicaciones en el análisis de alimentos, calidad del agua, vinos, explosivos y biofluidos para la detección de enfermedades.

Por otra parte, debido al importante número de datos recogido durante las medidas de lengua electrónica voltamétrica, resulta necesaria la aplicación de complejos algoritmos matemáticos para procesar la información. Herramientas de estadística multivariante tales como Análisis de Componentes Principales (PCA), Regresión de Mínimos Cuadrados Parciales (PLS) o Análisis Discriminante por PLS (PLS-DA) son frecuentemente aplicadas para la clasificación de las muestras (PCA) o para la generación de modelos predictivos para cuantificar (PLS) o clasificar las muestras (PLS-DA) (Martens, 2001). 
Dado el carácter aplicado de esta unidad didáctica, y el breve espacio de tiempo para que los alumnos puedan familiarizarse con el manejo de datos de sensores reales en el entorno industrial, la docencia de esta unidad didáctica se realiza con el uso de una herramienta informática intuitiva y de sencillo manejo como es el programa SOLO (Eigenvector Research, Inc.) y su aplicación a un conjunto de datos derivados de experimentos llevados a cabo previamente por el equipo de investigación del profesor. Como comprobación del dominio de la materia, a los alumnos se les requiere la reproducción del proceso en un nuevo conjunto de datos proporcionado por el profesor y obtenido también en el laboratorio por el equipo de investigación del docente. Para ambos conjuntos de datos existen los respectivos artículos de investigación publicados en revistas científicas con índice de impacto, en los que el alumno puede consultar con un mayor detalle el planteamiento del trabajo y la discusión de los resultados.

\section{Objetivos}

Los objetivos de esta unidad didáctica son los siguientes:

- Aproximación al concepto de lengua electrónica como estrategia de medida de bajo coste, sencillo funcionamiento y respuesta rápida, para la monitorización de la producción en el entorno industrial.

- Adquisición de nociones básicas de tratamiento de datos de gran dimensión derivados de lenguas electrónicas mediante estadística multivariante para cuantificación de parámetros en muestras reales, con el uso de un programa de estadística multivariante de uso sencillo e intuitivo.

- Uso de bases de bibliografía científica para la búsqueda de artículos científicos.

- Redacción de informes para la evaluación de las lenguas electrónicas aplicadas a los ejemplos consultados.

\section{Desarrollo de la innovación}

Para llevar a cabo la docencia de esta unidad didáctica se realizó en primer lugar en primer lugar por parte del docente una introducción al concepto de lengua electrónica, y a la estadística multivariante por medio de clases expositivas. El material sobre el que se realizó esta introducción estaba disponible para los alumnos en el aula virtual de la Universitat Politècnica de València (PoliformaT). En esta introducción se explicó también la dinámica de las sesiones de clases, los objetivos de la unidad y como se les iba a evaluar.

Una vez asentadas las bases teóricas imprescindibles, el docente procedió a presentar el programa que iba a utilizarse y la web desde donde cada alumno podría descargarse una versión de prueba gratuita del programa (Fig. 1), y que tendría validez por un tiempo suficiente ( 1 mes) como para realizar los análisis de datos durante las clases y en casa para realizar el trabajo con el que los alumnos debían ser evaluados. 


\section{$\lambda$ EIGENVECTOR}

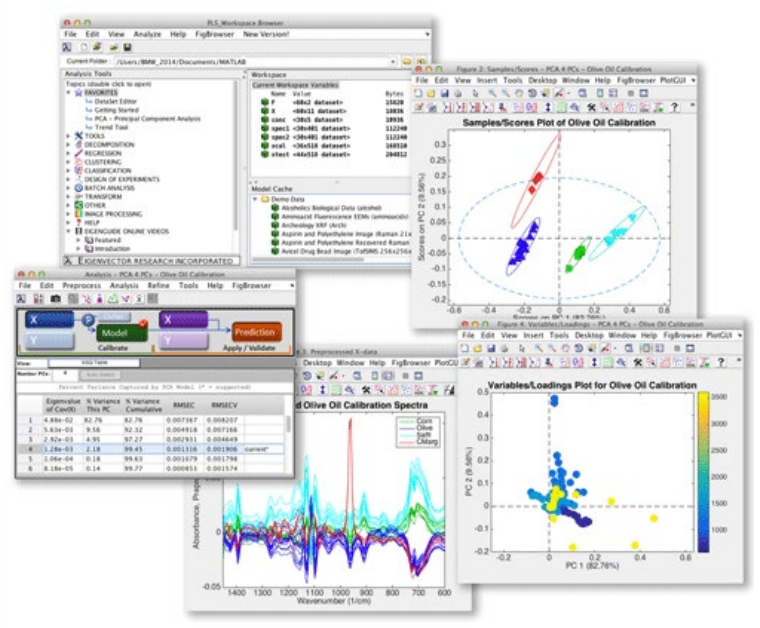

Fig. 1 Detalle de la página web del programa SOLO (Eingenvector Research, Inc) de análisis de estadística multivariante.

Los alumnos procedieron a descargar el programa y el docente les indicó los pasos a seguir con la instalación. Tras la instalación, el profesor les proporcionó dos conjuntos de datos procedentes de medidas de lengua electrónica voltamétrica. El primer conjunto de datos (medidas de ácidos orgánicos en disolución) serviría para trabajar con el programa en clase, y mostrar su funcionamiento a los alumnos. El segundo conjunto de datos (medidas de un herbicida en disolución) sería para reproducir el proceso de análisis de manera individualizada cada alumno, y serviría para evaluar el aprendizaje.

Los datos utilizados en este curso habían sido obtenidos por el equipo de investigación en el que colabora el docente, y su análisis había sido ya publicado en revistas científicas (ESCOBAR 2013, BATALLER 2012), por lo que el profesor, instruyó a los alumnos en la búsqueda de bibliografía científica en general, y en particular, para la búsqueda de publicaciones sobre sensores, lengua electrónica y su análisis estadístico (Fig. 2 y 3 ).

El profesor explicó la manera de dar formato a los datos de los sensores de manera que pudieran cargarse en el programa para su análisis. Del mismo modo, el profesor explicó a los alumnos la información necesaria que debía figurar en los ficheros sobre las muestras.

Con los datos de los ácidos orgánicos detectados mediante lengua electrónica cargados en la memoria del programa, el profesor indicó a los alumnos los pasos a seguir para realizar el estudio de PLS, ya que se pretendía cuantificar la cantidad de ácido ascórbico de las disoluciones. Explicadas las nociones básicas, el profesor explicó diferentes aproximaciones para simplificar el conjunto de datos, como son la selección de puntos, o incluso la selección de electrodos con objeto de reducir la dimensionalidad del conjunto de datos para reducir 
tiempo de cálculo. Por otra parte, dado que los datos provenían de diferentes electrodos, y si bien la filosofía de la lengua electrónica lleva implícita la unión de información cruzada, el profesor realizó un análisis de los electrodos de manera individual para estimar la bondad de cada uno de ellos para el análisis de las muestras.

Food Chemistry 138 (2013) 814-820

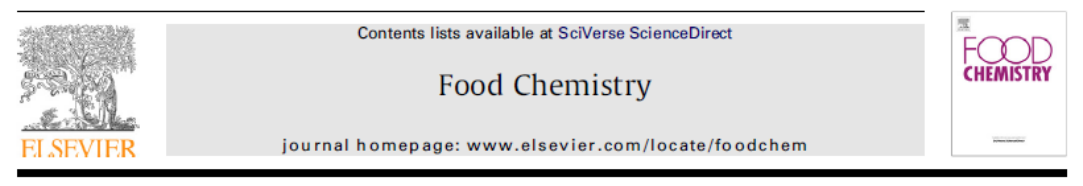

Analytical Methods

Quantification of organic acids using voltammetric tongues

Juan David Escobar ${ }^{\mathrm{a}, *}$, Miguel Alcaniz $^{\mathrm{b}}$, Rafael Masot ${ }^{\mathrm{b}}$, Ana Fuentes $^{\mathrm{a}}$, Roman Bataller ${ }^{\mathrm{b}}$, Juan Soto ${ }^{\mathrm{b}}$, Jose Manuel Barat ${ }^{a}$

2Departamento de Tecnología de Alimentos, Universitat Politècrica de Valìnda, Camino de Vera s/n, 46022 Valencia, Spain

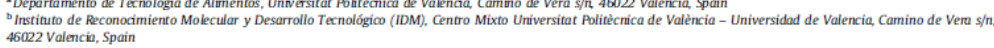

Fig2. Artículo de investigación cuyos datos fueron utilizados para explicar las nociones básicas del análisis mediante estadística multivariante de datos de lengua electrónica voltamétrica.

Una vez hechos los diferentes análisis, el profesor explicó los parámetros de calidad que debían tener en cuenta los alumnos para la interpretación de los ajustes $\left(\mathrm{R}^{2}\right.$, error medio en la predicción, pendiente y ordenada en el origen del ajuste).

La última sesión de la unidad se dedicó a la lectura e ingreso en el programa de estadística de los datos de glifosato para su posterior análisis mediante estadística multivariante (Fig. 3). Asimismo, el profesor repasó los conceptos que se habían estudiado en la unidad, de manera que los alumnos tuvieran reciente el procedimiento a seguir y pudieran resolver con el profesor las dudas que surgieran.

Sensors 2012, 12, 17553-17568; doi:10.3390/s121217553

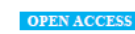

sensors

ISSN 1424-8220

Article

Glyphosate Detection by Means of a Voltammetric Electronic Tongue and Discrimination of Potential Interferents

Román Bataller ${ }^{1}$, Inmaculada Campos ${ }^{1,2,3}$, Nicolas Laguarda-Miro ${ }^{1,4}$, Miguel Alcañiz ${ }^{1,5}$, Juan Soto ${ }^{1,2, *}$, Ramón Martinez-Máñez ${ }^{1,2,3}$, Luis Gil ${ }^{1,5}$, Eduardo Garcia-Breijo ${ }^{1,5}$ and Javier Ibáñez-Civera ${ }^{1,5}$

1 Centro de Reconocimiento Molecular y Desarrollo Tecnológico (IDM), Unidad Mixta Universidad Politécnica de Valencia-Universidad de Valencia de Valéncia,

Camino de Vera s/n, E-46022 Valencia, Spain; E-Mail: robapra@upvnet.upv.es

2 Departamento de Quimica, Universidad Politécnica de Valencia, Camino de Vera s/n, E-46022 Valencia, Spain; E-Mails: incasan2@upvnet.upv.es (I.C.); jsotoca@upv.es (J.S.); rmaez@upv.es (R.M.M.)

CIBER de Bioingenieria, Biomateriales y Nano medicina (CIBER-BBN), Bellaterra, E-08193 Barcelona, Spain

Departamento de Ingenieria Quimica y Nuclear, Universidad Politécnica de Valencia,

Camino de Vera, s/n, E-46022 Valencia, Spain; E-Mail: milami@iqn upv.es

Departamento de Ingenieria Electrónica. Universidad Politécnica de Valencia. Camino de Vera, s/n.

E-46022 Valencia, Spain; E-Mails: mialcan@upvnet.upv.es (M.A.); 1gi1@eln.upv.es (L.G.); egarciab@eln upv.es (E.G.B.); jibanyez@eln upv.es (J.I.C.)

Fig 3. Artículo de investigación cuyos datos fueron utilizados por los alumnos para afianzar los conceptos y habilidades adquiridas y presentar un informe para su evaluación. 
Posteriormente el profesor dio a los alumnos un plazo para realizar los trabajos individuales con los datos de gliofosato, consultar dudas y enviar el análisis por correo electrónico o entregado impreso al profesor.

\section{Resultados}

Según se deduce del trabajo entregado, todos los alumnos adquirieron los conocimientos básicos necesarios para realizar el análisis crítico de los datos de lengua electrónica basada en voltametría, realizaron los análisis estadísticos y enviaron los trabajos. Aproximadamente la mitad de los alumnos envió los trabajos fuera del plazo inicialmente propuesto, si bien es cierto que los trabajos demostraron que los alumnos habían afianzado los conceptos. En los trabajos, los alumnos fueron capaces de reproducir parte de los resultados reflejados en las publicaciones científicas que se les proporcionaron como material de consulta. Adicionalmente, los alumnos aprendieron diversas estrategias para simplificar el conjunto de datos de partida para conseguir disminuir el tiempo de cálculo y simplificar los modelos estadísticos obtenidos, comprobando en sus datos como se podía llegar a un compromiso entre simplicidad del modelo e información requerida.

\section{Conclusiones}

La enseñanza de técnicas de análisis multivariante sobre datos reales en cursos de máster se puede realizar en el entorno docente e investigador, utilizando un software de sencillo manejo e intuitivo y analizando datos reales de experimentos realizados en el laboratorio del grupo de investigación. Todo ello en el contexto de la aplicación de estos sensores a la medida de parámetros en muestras de interés industrial. El tiempo de licencia de demostración fue suficiente para el desarrollo de las clases y posteriormente para que cada alumno realizara el trabajo en su casa.

\section{Referencias}

BATALlER, R. CAMPOS, I. LAGUARDA-MIRO, N. ALCAÑIZ, M. SOTO, J. MARTÍNEZ-MÁÑEZ, R. GIL, L. GARCÍA-BREIJO, E. IBÁÑEZ.CIVERA, J. (2012) "Glyphosate Detection by Means of a Voltammetric Electronic Tongue and Discrimination of Potential Interferents" Sensors, vol 12, p 17553-17568

ESCOBAR, J.D. ALCAÑIZ, M. MASOT, R. FUENTES, A. BATALLER, R. SOTO, J. BARAT J.M. (2013) "Quantification of organic acids using voltammetric tongues" Food Chemistry, vol 138, p 814-820

SOLO: PLS_Toolbox Solo 8.0, Eingenvector Research, Inc. Manson, WA USA 98831; software available at http://www.eigenvector.com.

MARTENS, M.(Magni) Martens (2001), Multivariate Analysis of Quality : an Introduction, Wiley.

WINQUIST, F, WIDE, P. LUNDSTRÖM, I. (1997). "An electronic tongue based on voltammetry" en Anal. Chim. Acta., vol. 357, p 21-31 